\title{
REVIEW
}

\section{Current and Novel Treatment Options for Metastatic Colorectal Cancer: Emphasis on Aflibercept}

\author{
Maria H. P. Dietvorst • Ferry A. L. M. Eskens
}

To view enhanced content go to www.biologicstherapy-open.com

Received: January 14, 2013 / Published online: February 28, 2013

(C) The Author(s) 2013. This article is published with open access at Springerlink.com

\section{ABSTRACT}

Worldwide, colorectal cancer (CRC) is the third most frequently diagnosed cancer in men and the second in women. Metastatic disease develops in more than half of the patients and carries a poor prognosis. Over the past three decades, significant advances have been made in the treatment of metastatic colorectal cancer (mCRC). The development of new cytotoxic agents and the incorporation of target-specific agents in first-, second-, third-, and nowadays even fourth-line treatment has prolonged median overall survival up to 24-28 months. However, 5-year survival rates remain disappointingly low. This review summarizes the currently available cytotoxic treatment

M. H. P. Dietvorst $(\bowtie) \cdot$ F. A. L. M. Eskens Department of Medical Oncology, Erasmus University Medical Center, Daniel den Hoed Cancer Center, Room HE120, PO BOX 2040, 3000 CA Rotterdam, The Netherlands e-mail: m.dietvorst@erasmusmc.nl

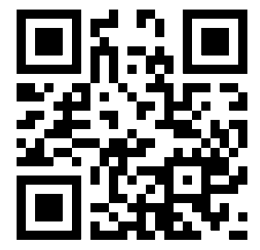

Enhanced content for this article is available on the journal web site: www.biologicstherapy-open.com options for mCRC, and highlights the further emerging role of vascular endothelial growth factor (VEGF)-inhibiting strategies, emphasizing the role of aflibercept. Aflibercept is a recombinant fusion protein with high VEGF affinity, and is the second antiangiogenic agent to obtain registration in the treatment of mCRC.

Keywords: Aflibercept; Cytotoxic therapy; Metastatic colorectal cancer; Oncology; Overall survival; Progression-free survival; Vascular endothelial growth factor inhibition

\section{INTRODUCTION}

Worldwide, colorectal cancer (CRC) is the third most prevalent cancer in men and the second in women. Globally, CRC has an estimated yearly incidence of more than 1.2 million and an annual mortality of over 600,000 [1]. Metastatic disease develops in $50 \%$ of patients and carries an ultimately fatal prognosis, with 5-year survival rates of only $10 \%$ [2].

Over the past three decades, significant progression has been made in the treatment of metastatic colorectal cancer (mCRC). Cytotoxic 
chemotherapy has changed from single-agent intravenous (i.v.) bolus 5-fluorouracil (5-FU) to a plethora of regimens containing 5-FU/ capecitabine, irinotecan, and/or oxaliplatin. In addition to these cytotoxic chemotherapies, the development and integration of so-called target-specific agents into the treatment of mCRC has further initiated a change in treatment paradigms and has profoundly shifted expectations in patients. By carefully selecting patients and combining and/or sequencing the currently available treatment options, median overall survival (OS) for mCRC patients has gradually increased from a median of 6 months to a median that is currently 24-28 months [3].

Unfortunately, however, until now no major gains have been made in 5-year survival rates and the number of patients cured from mCRC still remains disappointingly low.

Target-specific agents that have found their way into the treatment of $\mathrm{mCRC}$ can be divided into agents targeting the tumoral epidermal growth factor receptor (EGFR), and those targeting the vascular endothelial growth factor (VEGF) and its receptors (VEGFR) expressed on endothelial cells. In this review, the authors will not discuss EGFR inhibition strategies, but will focus on the role of VEGF inhibition.

The need for new and more active antiangiogenic agents in the treatment of mCRC is felt by many. Despite great promises observed in preclinical models, and interesting hints of biological and clinical activity observed in early clinical studies performed over a period of almost four decades, it seems fair to state that the overall added value of angiogenesis inhibition in the treatment of mCRC is modest at best. Studies combining first- and second-line cytotoxic treatment with VEGFR tyrosine kinase inhibitors have almost invariably yielded negative results, with no significant effects on progression-free survival (PFS) and OS [4, 5]. Combining firstand second-line cytotoxic treatment with bevacizumab has demonstrated improvements in PFS, but only a few studies have been published that demonstrated effects on OS $[6,7]$.

Recently, however, three studies have been published demonstrating the effects of angiogenesis inhibition on OS, and the publication of two of these studies has boosted recent US Food and Drug Administration (FDA) registration of regorafenib and aflibercept [8-10]. This landmarked the second and third registration of an antiangiogenic agent in the treatment of mCRC after the first registration of such an agent took place almost a decade ago.

The Treatment across Multiple Lines (TML) trial provided statistical evidence that continuing bevacizumab treatment beyond tumor progression (while changing the chemotherapy schedule) improves OS [8]. The CORRECT trial showed that regorafenib, which is a broad-spectrum tyrosine kinase inhibitor with antiangiogenic activity, was also associated with a statistical improvement in OS in patients with mCRC who progressed after all standard treatment options [9]. Finally, the VELOUR trial demonstrated a statistically significant improvement in OS in patients when given second-line treatment with infusional 5-FU/leucovorin and irinotecan (FOLFIRI) and aflibercept [10].

After a brief summary of the currently available cytotoxic treatment options for mCRC and the role of bevacizumab within these options, this review describes the structure and function of aflibercept, and highlights the results of early clinical studies. After discussing the final results of the VELOUR study and the impact of treating mCRC with 
VEGF inhibitors beyond progression, a brief discussion about the role of aflibercept within mCRC treatment is given.

\section{METHODS}

A systematic literature search was conducted using PubMed with no limitations of language or years of publication. The following keywords were used: "metastatic colorectal cancer," "aflibercept," "bevacizumab," "cytotoxic therapy," and "vascular endothelial growth factor." Additionally, the authors reviewed abstracts related to aflibercept from major congresses in North America and Europe over the last 5 years.

\section{CURRENTLY AVAILABLE CYTOTOXIC TREATMENT OPTIONS FOR $\mathrm{mCRC}$}

As mentioned previously, fluoropyrimidines have for decades been the backbone of systemic treatment of mCRC. 5-FU functions as a nucleoside analog, preventing DNA replication, and leucovorin potentiates its effect [11]. Capecitabine is the oral prodrug of 5-FU and has demonstrated efficacy at least similar to that of i.v. bolus 5-FU $[12,13]$.

Irinotecan, a topoisomerase I inhibitor, was initially approved for second-line treatment of mCRC in 1998 based upon results from two randomized trials demonstrating an improvement in both PFS and OS when compared to best supportive care or 5-FU/ leucovorin in patients with mCRC refractory to 5-FU [14, 15]. Nowadays, irinotecan containing combination schedules are considered an essential part of standard firstand second-line treatments of mCRC.

Oxaliplatin, a platinum derivative, formed another milestone in mCRC treatment. Two randomized trials evaluated the addition of oxaliplatin to a regimen of 5-FU/leucovorin in first-line therapy for mCRC. The results showed a higher response rate, increased PFS, but had no significant effect on OS $[16,17]$. A phase 3 trial demonstrated efficacy of oxaliplatin in addition to 5-FU/leucovorin as second-line therapy for mCRC [18]. In 2002, these data led to the approval of oxaliplatin for treatment in mCRC, and since then oxaliplatin is considered another essential part of treatment strategies in this disease [19].

Numerous different combinations of i.v. or oral 5-FU with irinotecan and/or oxaliplatin in schedules, such as infusional 5-FU/leucovorin and oxaliplatin (FOLFOX), capecitabine and oxaliplatin (CAPOX/XELOX), FOLFIRI, and infusional 5-FU/leucovorin, oxaliplatin, and irinotecan (FOLFOXIRI), have convincingly shown their effectiveness in first- and/or second-line treatment settings of mCRC [20]. Although upfront combination therapy is frequently used, especially in patients with symptomatic disease or in patients in whom a response is required to open possibilities for additional treatment, such as surgery, exposure to all mentioned cytotoxic drugs throughout the sequential lines of treatment of mCRC appears to be as justifiable as a specific combination of therapies at a particular moment [21].

\section{ANTIANGIOGENIC AGENTS IN mCRC: BEVACIZUMAB}

Bevacizumab is a humanized monoclonal antibody that binds to VEGF. Bevacizumab prevents binding of circulating VEGF-A to specific endothelial VEGFRs, which results in the inhibition of VEGF signalling and angiogenesis [22]. 
In first-line treatment of mCRC, bevacizumab has not demonstrated singleagent activity, but when combined with various chemotherapy regimens, bevacizumab almost invariably increases response rates and PFS [23]. Of note, bevacizumab showed impact on OS in only one randomized study, which used the 5-FU/leucovorin and irinotecan (IFL) regimen containing bolus 5-FU and irinotecan administration as a backbone [6]. This IFL schedule is generally considered to be less active than infusional 5-FU schedules.

In second-line treatment of mCRC, the E3200 study demonstrated improvement in OS of bevacizumab when combined with FOLFOX [7]. In this study, bevacizumab was dosed at $10 \mathrm{mg} / \mathrm{kg}$ every 14 days.

These two trials led to the FDA approval of bevacizumab in 2004 for first-line (and, in 2006, for second-line) treatment of mCRC when combined with 5-FU-containing regimens.

In addition to these randomized trials, the observational Bevacizumab Regimens: Investigation of Treatment (BRiTE) and Avastin Registry: Investigation of Efficacy and Safety (ARIES) studies suggest that the use of bevacizumab could be effective after progression in first-line mCRC treatment $[24,25]$. In a multivariate analysis, the sustained inhibition of angiogenesis was found to be associated with improved survival. However, findings from observational studies may be prone to bias and, therefore, should be interpreted with great caution.

The recently published TML study is the first prospective randomized trial evaluating the role of continuing bevacizumab as part of secondline treatment for patients with mCRC who progressed after first-line bevacizumabcontaining therapy [8]. In this trial, 820 patients who had received first-line bevacizumab-containing chemotherapy were randomized to receive second-line chemotherapy with alternate cytotoxics with or without bevacizumab. The primary endpoint of this study was OS, and the secondary endpoint was PFS. Median OS was 11.2 months for chemotherapy plus bevacizumab and 9.8 months for chemotherapy alone [hazard ratio (HR) $0.81 ; 95 \%$ confidence interval (CI) 0.69-0.94; $P=0.0062]$. Median PFS was 5.7 months and 4.1 months, respectively (HR $0.68 ; 95 \%$ CI $0.59-0.78 ; \quad P<0.0001)$. These results were regardless of whether oxaliplatin or irinotecan had been part of the second-line treatment.

A second phase 3 clinical trial with a similar design stopped accrual when results of the TML study were released [26]. Results from this study, however, also showed that the continuation of bevacizumab beyond progression in patients with mCRC resulted in a significant benefit in PFS.

\section{ANTIANGIOGENIC AGENTS IN mCRC: AFLIBERCEPT}

Aflibercept (formerly known as VEGF-trap or AVE0005) is a novel human recombinant protein which is constructed by fusing the second extracellular domain of VEGFR-1 and the third extracellular domain of VEGFR-2 to the Fc segment of immunoglobulin G1. It functions as a soluble, high-affinity decoy receptor preventing VEGF-A, VEGF-B, and placental growth factor (PIGF) from interacting with their cognate receptors [27]. In contrast to bevacizumab, aflibercept not only targets VEGFA, but also VEGF-B and PlGF, forming a pharmacologic blockade of the VEGF pathway. Preclinical studies in various tumor models have demonstrated reduction of tumor vasculature and size, and inhibition of ascites formation [28]. 


\section{Clinical Trials with Aflibercept}

\section{Phase 1}

Completed phase 1 trials suggest that singleagent aflibercept is well tolerated. The first phase I study evaluated safety, pharmacokinetics, and pharmacodynamics of i.v.-administered aflibercept in 47 patients with a variety of refractory solid tumors and nonHodgkin's lymphoma [29]. The most common adverse events were fatigue, nausea, and vomiting, and their frequency was not dose-dependent. Toxicities associated with aflibercept included dysphonia, hypertension, and proteinuria. Dose-limiting toxicities were rectal ulceration and proteinuria at the $7.0 \mathrm{mg} /$ $\mathrm{kg}$ dose. The recommended dose was set at $4 \mathrm{mg} / \mathrm{kg}$ i.v. every 2 weeks [29].

A dose-escalation study of subcutaneousadministered aflibercept in 38 patients with advanced solid tumors revealed proteinuria, fatigue, injection-site reaction, nausea, myalgia, anorexia, hypertension, and hoarseness as the most frequently occurring side effects [30]. Grade 3/4 hypertension and proteinuria occurred in 8 and $3 \%$ of patients, respectively, and was reversible. Due to solubility and dosing limits with the subcutaneous formulation, the maximum tolerated dose could not be determined.

In a phase 1 dose-escalation study of i.v. aflibercept in combination with irinotecan, infusional 5-FU, and leucovorin (LV5FU2) in 38 patients with advanced solid tumors, the recommended dose of aflibercept could also be set at $4 \mathrm{mg} / \mathrm{kg}$ i.v. every 2 weeks [31]. The most common grade $3 / 4$ adverse events with the $4 \mathrm{mg} / \mathrm{kg}$ dose were neutropenia, hypertension, fatigue, and diarrhea. Antitumor activity was assessed in 10 evaluable patients receiving aflibercept at the $4 \mathrm{mg} / \mathrm{kg}$ dose. Five patients had partial responses (three colorectal cancer, one pancreatic cancer, one synovial carcinoma) and five patients had stable disease lasting more than 3 months [31].

\section{Phase 2}

A multicenter, open-label, phase 2 clinical trial investigated aflibercept in patients with previously treated mCRC [32]. In total, 75 patients were enrolled in two cohorts, bevacizumab naïve $(n=24)$ or bevacizumab treated $(n=51)$. Single-agent aflibercept was given at the dose of $4 \mathrm{mg} / \mathrm{kg}$ i.v. every 2 weeks. In the bevacizumab-naïve cohort, the best response was stable disease for 16 weeks or more in five patients. In the prior bevacizumab cohort, one patient achieved a partial response and six patients had stable disease for 16 weeks or more. The median PFS in the bevacizumab-naïve and prior bevacizumab cohorts was 2.0 months (95\% CI 1.7-8.6 months) and 2.4 months (95\% CI 1.9-3.7 months), respectively. The most common grade 3 or higher treatment-related adverse events were hypertension, proteinuria, fatigue, and headache. It was concluded that aflibercept was well tolerated and had limited single-agent activity in pretreated mCRC, regardless of prior therapy with bevacizumab.

The AFFIRM trial is a randomized phase 2 study investigating aflibercept combined with modified FOLFOX6 (infusional 5-FU/leucovorin and oxaliplatin) in first-line treatment of mCRC [33]. The primary endpoint of this study was PFS rate at 12 months. The secondary outcome measures included overall response rate, PFS, and OS. Preliminary results show no significant difference in PFS at 1 year.

\section{Phase 3}

The phase 3 VELOUR trial was a randomized, placebo-controlled study evaluating the combination of aflibercept plus FOLFIRI in the 
second-line treatment of mCRC after oxaliplatin failure [10]. In this trial 1,226 patients were randomly assigned to receive either aflibercept (4 mg/kg i.v.; 612 patients) or placebo (614 patients) every 2 weeks in combination with FOLFIRI. Approximately one third of these patients had previously been treated with bevacizumab. Addition of aflibercept to FOLFIRI significantly improved OS from 12.06 months to 13.50 months, respectively (HR $0.817 ; \quad 95.34 \% \quad$ CI 0.713-0.937; $\quad P=0.0032$ ). Aflibercept also significantly improved PFS and response rate. Median PFS was 6.90 months in the aflibercept arm and 4.67 months in the control arm (HR $0.758 ; \quad 95 \%$ CI $0.661-0.869 ; \quad P<0.0001)$. Response rate was $19.8 \% \quad(95 \%$ CI 16.4-23.2\%) with aflibercept plus FOLFIRI versus $11.1 \% \quad(95 \% \quad$ CI $\quad 8.5-13.8 \%)$ with placebo plus FOLFIRI $(P=0.0001)$. Grade 3 and 4 adverse events with at least a $2 \%$ higher incidence in the aflibercept arm versus placebo were diarrhea, asthenic conditions, stomatitis and ulceration, infections, hypertension, gastrointestinal and abdominal pain, neutropenia and neutropenic complications, and proteinuria. Adverse events leading to treatment discontinuation occurred in $26.8 \%$ of patients receiving aflibercept plus FOLFIRI and $12.1 \%$ of patients receiving placebo plus FOLFIRI. The adverse events leading most frequently to permanent discontinuation were asthenic conditions, infections, diarrhea, and hypertension. Compared with the TML study and the AVastin IRInotecan (AVIRI) study (bevacizumab plus FOLFIRI in first-line mCRC [34]), the VELOUR trial demonstrated a similar toxicity profile. In the VELOUR trial, a prespecified subgroup analysis demonstrated no significant interaction of prior bevacizumab therapy on PFS and OS in both treatment arms. These results suggest that aflibercept is effective for patients with mCRC who progressed after first-line bevacizumab-containing therapy.

In the near future, a randomized study of aflibercept is planned for patients with mCRC who have received perioperative FOLFOX chemotherapy and have subsequently undergone complete resection or ablation of metastatic disease [35].

\section{CONCLUSION}

In 2012, aflibercept obtained FDA approval for the second-line treatment of mCRC in combination with FOLFIRI chemotherapy. The approval of a second antiangiogenic agent for this indication is an important milestone in the treatment of this disease. Aflibercept has a powerful biologic profile, targeting VEGF-A, VEGF-B, and PIGF, and the pivotal VELOUR study provided compelling evidence that aflibercept improves OS in second-line mCRC, which, so far, is an almost unique feature [10].

Last year, more relevant results were published in the setting of mCRC treatment, as in addition to the VELOUR data, the TML study demonstrated that bevacizumab continuation beyond progression of first-line mCRC treatment also yielded prolongation of OS [8]. When taking a close look at the data of these two landmark trials, realizing that a formal head-to-head comparison cannot be made, the similarity between the data is striking. Both studies show a 1.4-month benefit in OS, whereas the toxicity profile of bevacizumab and aflibercept shows an almost complete overlap. Another conclusion from this, and again emphasizing the indirect comparison between these studies, is that most likely the sequence of first- and secondline chemotherapy does not seem to be very distinctive in determining the clinical endpoint of survival. 
Whereas bevacizumab has demonstrated activity in various studies of first-line treatment of mCRC, at this moment only preliminary data from a randomized phase 2 (AFFIRM) trial combining aflibercept with modified FOLFOX in first-line mCRC are available. Therefore, making any comparisons regarding the activity of these two agents in first-line treatment of mCRC is, at best, currently speculative.

Given the seemingly comparable efficacy of bevacizumab and aflibercept in $\mathrm{mCRC}$, it is highly questionable, in the authors' view, whether a direct comparison between these two antiangiogenic agents combined with chemotherapy within the context of a large randomized, noninferiority study in first-line treatment will ever be pursued.

The promising results with aflibercept demonstrate that the concept of antiangiogenesis inhibition remains a valuable area of investigation, even years after the registration of bevacizumab. This new drug represents an important addition to the armamentarium of mCRC treatment.

However, and to put the data into a critical perspective, current antiangiogenic therapies are effective to only a limited degree in a small, poorly-predictable subset of patients with mCRC.

Additional studies, therefore, will be necessary to determine the exact role of aflibercept in various subsets of patients, and hopefully these future trials will reveal optimal combinations with all agents currently available. This clinical research will have to be focussed on the recognition and/or development of biomarkers that may help to select those patients most likely to benefit from angiogenesis inhibition. Reliable markers can then predict the response and ultimately prognosis of patients, and will as such provide the foundation for an optimally individualized/ personalized treatment of mCRC.

\section{ACKNOWLEDGMENTS}

Dr. Dietvorst is the guarantor for this article, and takes responsibility for the integrity of the work as a whole.

Conflict of interest. Dr. Dietvorst has no personal, commercial, academic, or financial conflicts of interest to declare. Dr. Eskens has no personal, commercial, academic, or financial conflicts of interest to declare. There was no funding or sponsorship received in relation to this paper.

Open Access. This article is distributed under the terms of the Creative Commons Attribution Noncommercial License which permits any noncommercial use, distribution, and reproduction in any medium, provided the original author(s) and the source are credited.

\section{REFERENCES}

1. Jemal A, Bray F, Center MM, Ferlay J, Ward E, Forman D. Global cancer statistics. CA Cancer J Clin. 2011;61:69-90.

2. Cidón EU. The challenge of metastatic colorectal cancer. Clin Med Insights Oncol. 2010;4:55-60.

3. Chu E. An update on the current and emerging targeted agents in metastatic colorectal cancer. Clin Colorectal Cancer. 2012;11:1-13.

4. Hoff PM, Hochhaus A, Pestalozzi BC, et al. Cediranib plus FOLFOX/CAPOX versus placebo plus FOLFOX/CAPOX in patients with previously untreated metastatic colorectal cancer: a randomized, double-blind, phase III study (HORIZON II). J Clin Oncol. 2012;30:3596-603.

5. Van Cutsem E, Bajetta E, Valle J, et al. Randomized, placebo-controlled, phase III study of oxaliplatin, fluorouracil, and leucovorin with or without 
PTK787/ZK 222584 in patients with previously treated metastatic colorectal adenocarcinoma. J Clin Oncol. 2011;29:2004-10.

6. Hurwitz H, Fehrenbacher L, Novotny W, et al. Bevacizumab plus irinotecan, fluorouracil, and leucovorin for metastatic colorectal cancer. N Eng J Med. 2004;350:2335-42.

7. Giantonio BJ, Catalano PJ, Meropol NJ, et al. Eastern Cooperative Oncology Group Study E3200. Bevacizumab in combination with oxaliplatin, fluorouracil, and leucovorin (FOLFOX4) for previously treated metastatic colorectal cancer: results from the Eastern Cooperative Oncology Group Study E3200. J Clin Oncol. 2007;25:1539-44.

8. Bennouna J, Sastre J, Arnold D, ML18147 Study Investigators, et al. Continuation of bevacizumab after first progression in metastatic colorectal cancer (ML18147): a randomized phase 3 trial. Lancet Oncol. 2013;14:29-37.

9. Grothey A, Van Cutsem E, Sobrero A, CORRECT Study Group, et al. Regorafenib monotherapy for previously treated metastatic colorectal cancer (CORRECT): an international, multicentre, randomised, placebo-controlled, phase 3 trial. Lancet. 2012;381:303-12.

10. Van Cutsem E, Tabernero J, Lakomy R, et al. Addition of aflibercept to fluorouracil, leucovorin, and irinotecan improves survival in a phase III randomized trial with metastatic colorectal cancer previously treated with an oxaliplatin-based regimen. J Clin Oncol. 2012;30:3499-506.

11. Longley DB, Harkin DP, Johnston PG. 5-fluorouracil: mechanisms of action and clinical strategies. Nat Rev Cancer. 2003;3:330-8.

12. Van Cutsem E, Twelves C, Cassidy J, Xeloda Colorectal Cancer Study Group, et al. Oral capecitabine compared with intravenous fluorouracil plus leucovorin in patients with metastatic colorectal cancer: results of a large phase III study. J Clin Oncol. 2001;19: 4097-106.

13. Hoff PM, Ansari R, Batist G, et al. Comparison of oral capecitabine versus intravenous fluorouracil plus leucovorin as first-line treatment in 605 patients with metastatic colorectal cancer: results of a randomized phase III study. J Clin Oncol. 2001;19:2282-92.

14. Cunningham D, Pyrhönen S, James RD, et al. Randomised trial of irinotecan plus supportive care versus supportive care alone after fluorouracil failure for patients with metastatic colorectal cancer. Lancet. 1998;352:11413-8.
15. Rougier $\mathrm{P}$, van Cutsem E, Bajetta $\mathrm{E}$, et al. Randomised trial of irinotecan versus fluorouracil by continuous infusion after fluorouracil failure in patients with metastatic colorectal cancer. Lancet. 1998;352:1407-12.

16. Giachetti S, Perpoint B, Zidani R, et al. Phase III multicenter randomized trial of oxaliplatin added to chronomodulated fluorouracil-leucovorin as first-line treatment of metastatic colorectal cancer. J Clin Oncol. 2000;18:136-47.

17. De Gramont A, Figer A, Seymour $M$, et al. Leucovorin and fluorouracil with or without oxaliplatin as first-line treatment in advanced colorectal cancer. J Clin Oncol. 2000;18:2938-47.

18. Rothenberg ML, Oza A, Bigelow $\mathrm{RH}$, et al. Superiority of oxaliplatin and 5-FU/leucovorin over either therapy alone in patients with progressive colorectal cancer following irinotecan and 5-FU/leucovorin: interim results of a phase III trial. J Clin Oncol. 2003;21:2059-69.

19. Stein A, Arnold D. Oxaliplatin: a review of approved uses. Expert Opin Pharmacother. 2012;13:125-37.

20. Ficorella C, Bruera G, Cannita K, et al. Triplet chemotherapy in patients with metastatic colorectal cancer: toward the best way to safely administer a highly active regimen in clinical practice. Clin Colorectal Cancer. 2012;11:229-37.

21. Grothey A, Sargent D, Goldberg RM, Schmoll HJ. Survival of patients with advanced colorectal cancer improves with the availability of fluorouracilleucovorin, irinotecan, and oxaliplatin in the course of treatment. J Clin Oncol. 2004;22:1209-14.

22. McRee AJ, Goldberg RM. Optimal management of metastatic colorectal cancer. Current status. Drugs. 2011;71:869-84.

23. Strickler JH, Hurwitz HI. Bevacizumab-based therapies in the first-line treatment of metastatic colorectal cancer. Oncologist. 2012;17:513-24.

24. Grothey A, Sugrue MM, Purdie DM, et al. Bevacizumab beyond first progression is associated with prolonged overall survival in metastatic colorectal cancer: results from a large observational cohort study (BRiTE). J Clin Oncol. 2008;26:5326-34.

25. Cohn AL, Bekaii-Saab T, Bendell JC, et al. Clinical outcomes in bevacizumab (BV)-treated patients (pts) with metastatic colorectal cancer (mCRC): results from ARIES observational cohort study (OCS) and confirmation of BRiTE data on BV beyond progression (BBP). J Clin Oncol. 2010;28(Suppl.):Abstract 3596. 
26. Masi G, Loupakis F, Allegrini G, et al. A randomized phase III study evaluating the continuation of bevacizumab (BV) beyond progression in metastatic colorectal cancer (mCRC) patients (pts) who received BV as part of first-line treatment: results of the BEBYP trial by the Gruppo Oncologico Nord Ovest (GONO). Ann Oncol. 2012;23(Suppl. 9):LBA 17.

27. Wang T-F, Lockhart AC. Aflibercept in the treatment of metastatic colorectal cancer. Clin Med Insights Oncol. 2012;6:19-30.

28. Gaya A, Tse V. A preclinical and clinical review of aflibercept for the management of cancer. Cancer Treat Rev. 2012;38:484-93.

29. Lockhart AC, Rothenberg ML, Dupont J, et al. Phase I study of intravenous vascular endothelial growth factor trap, aflibercept, in patients with advanced solid tumors. J Clin Oncol. 2009;28:207-14.

30. Tew WP, Gordon M, Murren J, et al. Phase 1 study of aflibercept administered subcutaneously to patients with advanced solid tumors. Clin Cancer Res. 2010;16:358-66.

31. Van Cutsem E, Khayat D, Verslype C, et al. Phase I dose-escalation study of intravenous aflibercept administered in combination with irinotecan, 5-fluorouracil and leucovorin in patients with advanced solid tumours. Eur J Cancer. 2013; 49:17-24.

32. Tang PA, Cohen SJ, Kollmannsberger C, et al. Phase II clinical and pharmacokinetic study of aflibercept in patients with previously treated metastatic colorectal cancer. Clin Cancer Res. 2012;18: 6023-31.

33. Pericay C, Folprecht G, Saunders M, et al. Phase 2 randomized, noncomparative, open-label study of aflibercept and modified Folfox6 in the first-line treatment of metastatic colorectal cancer (AFFIRM). Ann Oncol. 2012;23(Suppl. 4):iv16.

34. Sobrero A, Ackland S, Clarke S, et al; AVIRI Trial Investigators. Phase IV study of bevacizumab in combination with infusional fluorouracil, leucovorin and irinotecan (FOLFIRI) in first-line metastatic colorectal cancer. Oncology. 2009;77: 113-9.

35. Adjuvant Aflibercept for Metastatic Colorectal Cancer (C261). Clinical Trials.gov website. Available at: http://clinicaltrials.gov/show/NCT016 69720. Accessed Feb 42013. 